\title{
A Smart Decision Making System for the Selection of Production Parameters using Digital Twin and Ontologies
}

\author{
ABADI Mohammed ${ }^{1}$, BEN-AZZA Hussain ${ }^{4}$ \\ Laboratory of Mechanics, Mechatronics and Command \\ Modeling, Information Processing and Control of Systems \\ (MTICS) Team \\ Ecole Nationale Supérieure d'Arts et Métiers (ENSAM- \\ Meknès), Moulay Ismail University, B.P. 4042, 50000 \\ Meknes, Morocco
}

\author{
ABADI Chaimae ${ }^{2}$ \\ Laboratory of Mechanics, Mechatronics and Command \\ Electric Power, Maintenance and Innovation Team, Ecole \\ Nationale Supérieure d'Arts et Métiers (ENSAM-Meknès) \\ Moulay Ismail University, B.P. 4042, 50000 \\ Meknes, Morocco
}

\author{
ABADI Asmae ${ }^{3}$ \\ INSA, Euro-Mediterranean University of Fez \\ Fez, Morocco
}

\begin{abstract}
Currently, the industrial and economic environment is highly competitive, forcing companies to keep up with technological progress and to be efficient in terms of quality and responsiveness, not only to survive, but also to dominate the market. So, to achieve this goal, companies are always looking to master their production processes, as well as to enlarge their range of products, either by developing new products or by improving old ones. This confronts companies to many problems, including the identification of adequate and optimal production parameters for the development of their products. In this context, a decision making system based on digital twins (DT), case-based reasoning (CBR) and Ontologies is proposed. The originality of this work lies in the fact that it combines three emerging artificial intelligence tools for modeling, reasoning and decision making. Thus, this work proposes a new flexible and automated system that ensures an optimal selection of production parameters for a given complex product. An industrial case of study is developed to illustrate the effectiveness of the proposed approach.
\end{abstract}

Keywords-Production parameters selection; digital twin; casebased reasoning; ontologies; automation; cyber-physical systems; decision making; artificial intelligence

\section{INTRODUCTION}

Nowadays, the industrial environment is continuously changing and the industrial competition has become more and more severe due to the consumers exigencies which have become more and more complex and highly personalized. On the one hand, in order to deal with these changes, companies are trying to have flexible manufacturing systems [1] that will allow them to diversify their products and respond to market demands. This diversification requires an efficient choice of the production parameters, in order to realize quality products, with optimal production costs and in the best delays. But in the majority of cases, this choice is made manually, and therefore requires important expenses in terms of time and money. On the other hand, companies tend to make their manufacturing systems intelligent and in real-time [2] to have faster and more efficient production processes. Thus, new concepts have been introduced to the industrial environment namely Digital Twin technologies and manufacturing intelligence [3]. It is in this context that this document has been developed. Indeed, an approach is proposed to automate the choice of production parameters. This approach is resulting from the combination of three artificial intelligence tools, namely:

- Digital Twins (DTs): are an artificial intelligence tool that is capable of copying the operation of production systems in real time and analyzing them, by ensuring a reciprocal interaction between the physical entities and their virtual counterparts [4].

It will allow us to simulate the production process and its parameters that will be automatically computed using the other components of the proposed SPPDT system. The Digital Twin will ensure this simulation in real time and will allow validating the production parameters on the virtual production process before its physical implementation.

- Case-based reasoning (CBR): is one of the tools of artificial intelligence that is based on the use of old functional and efficient solutions to problems encountered in order to treat and solve new similar problems faced $[5,6]$.

The role of CBR in the proposed approach is to ensure a part of the reasoning and decision-making support.

- Ontologies: a set of concepts and parameters that characterize a specialized domain (for example: the pharmaceutical industry) [7], it allows to define the meaning of words (synonyms, thesaurus, ...) and to exchange this information in a unique format/language. 
The use of ontologies will ensure interoperability between the different elements of the cyber-physical model of the digital twin. Their use will also ensure the expressiveness of the treated information and preserve their semantics. In addition to that, ontologies will ensure reasoning and decision making for the selection of optimal production parameters.

So, the first section of this paper presents a literature review on the main concepts used for the development of the proposed approach SPPDT (Selection of Production Parameters based on the Digital Twin) namely the digital twins and the ontologies. The second section describes in a general way the proposed approach SPPDT and the functioning of its system. In the following sections, the different modules of the proposed approach are explained in detail, particularly their roles and their functioning. Finally, in the last section, a case study is illustrated in order to prove the good functioning and the efficiency of the developed approach.

\section{RELATED WORK}

We review in this section the main concepts combined in this work, which are Digital Twins and Ontologies.

\section{A. Digital Twin Concept}

The digital twin has become one of the most frequently used tools for managing problems in cyber-physical systems [8]. This concept appeared for the first time at the end of the 1960s, as part of the Nasa Apollo project. This project consisted in the creation of two similar space vehicles: one is sent on a mission and "its twin" is left on earth to follow its state. And so at that time, there was talk about a physical twin that represents the real operating conditions for the simulation of the behavior in real time.

Afterwards, in one of his presentations on product lifecycle management in 2003 at the University of Michigan, Michael Grieves went from this physical model to present a new conceptual model named "mirror space model", and later named "information mirror model" [9]. This one is used to represent virtually and numerically a physical product. Then, in their white paper on the origins of the digital twin, Michael Grieves and John Vickers proposed a general structure for the digital twin that consists of three basic parts $[10,11,12]$.

- Physical entity: It usually contains various subsystems that have as role the execution of predefined tasks during operation. In addition to that, these subsystems are equipped with a variety of sensors that collect the necessary information about the working conditions of these subsystems.

- Virtual model: It is a model that reproduces all the characteristics of the physical entity to its geometric and dimensional specifications, its physical properties such as construction materials, the instructions necessary for the correct operation as well as the rules to be applied.

- Connection model: This is the link or interface between the two physical and virtual spaces. It is done through different technologies, including network communication, IoT and network security.
However, according to $[11,13]$, this structure of the digital twin previously proposed was not complete, and therefore two other dimensions were added to it, namely:

- Services: they can be decomposed into two classes. The first one is a class of business service which schematizes in a simplified and standardized way the inputs and outputs through software interfaces. While the second class is the functional service that ensures the conversion of data and algorithms into support services for the proper functioning of the DT.

- DT data model: It includes the data of all the other elements of the structure, namely the two physical and virtual spaces, the data of the services. In addition to that, it is enriched by the knowledge of the working domain and the merged data of both physical and virtual aspects.

\section{B. Comparing Approaches of Solving Interoperability Problems}

According to the ISO-14258-1998 standard, three approaches to achieve interoperability exist, including [14]:

- Integration: It consists in proposing a common standard data model between the different actors of the network. However, the level of compatibility achieved is limited because it is difficult to propose a consensus that is ideally adequate to each of the actors [15].

- Unification: Its principle is based on establishing direct semantic links between the different actors of the network. The problem is that after any modification of a network element, an update must be made at the level of the main model [16].

- Federation: It is based on the idea that each "business" must be able to maintain its own information model to guarantee its meaning and flexibility [15]. Therefore, it is based on logic in order to establish automatic connections between the models used to exchange information between the different actors.

In our context, it is essential to preserve the semantics of the considerable quantity of data that must be exchanged, as well as to ensure a high degree of exchange flexibility manifested in the rapidity and efficiency of decision-making at the right time.

Thus, the federation-based approach is the most suitable to accomplish these objectives, especially since it has become attainable due to information and communication technologies, which propose new modeling paradigms based on the use of ontologies.

\section{Overview on Ontologies}

According to the ancient Greek, the term "ontology" is composed of two words: "ontos" which means "to be" and the word "logos" which means "discourse". Then, the definitions of ontology have become various.

In fact, an ontology according to [17], is a characterization that is based on the creation of several axioms that describe the properties of concepts, individuals and relations existing in a 
domain of interest, in order to avoid ambiguities of understanding. In the recent decades, Ontologies have been widely exploited in many industrial applications, including knowledge representation [18], and the resolution of interoperability problems [15]. Indeed, the effectiveness of inference Ontologies in handling and solving interoperability problems lies in their ability to [19]:

- Integration and completeness, provided by the expressiveness of the language.

- Embedded intelligence, due to the reasoning capabilities of logical description languages.

- Dynamism and flexibility, due to the queries and web services.

According to [18], these uses of ontologies are possible as a result of its sustained expressiveness and reasoning capabilities.

- Expressiveness: the information treated by ontologies must be expressed with formal languages (a syntax, semantics and rules), in order to be understood by machines. For this reason, the Web Ontology Language (OWL) has been designated as a standard ontology language, recommended by the W3C [20]. Indeed, OWL offers a great capacity to define concepts and relations, based on the description logics (negation, restriction, existence of concepts and even intersection).

- Reasoning: New implicit information can be inferred about instances from the concepts, relations, axioms and rules explicitly defined in the ontology. In fact, ontologies are based on logic and therefore can perform inference operations using inference engines or reasoners.

Thus, in this article, the use of ontologies will play a crucial role, since it will ensure the interoperability between the different elements of the cyber-physical model of the digital Twin, the expressiveness of the treated information and the preservation of their semantics, as well as the reasoning and the help to the decision making.

\section{The Global Proposed SPPDT METHOdOLOGY}

The main objective of this article is the automatization of decision making for optimal selection of production parameters. In order to do that, a structured methodology based on Digital Twins (DT), Case Based Reasoning (CBR) and Ontologies is proposed. Indeed, the fusion of these three tools gives birth to SPPDT (selection of production parameters based on the digital twin) methodology.

As shown in Fig. 1, it is composed of two phases: construction phase and operation phase.

The first one (construction phase) contains two modules:

- The Data Collection Module (DCM): This first step is crucial in the proposed methodology. In fact, it is essential to collect the necessary information, either on the product or on the production parameters, which will be useful to make a complete and efficient study.

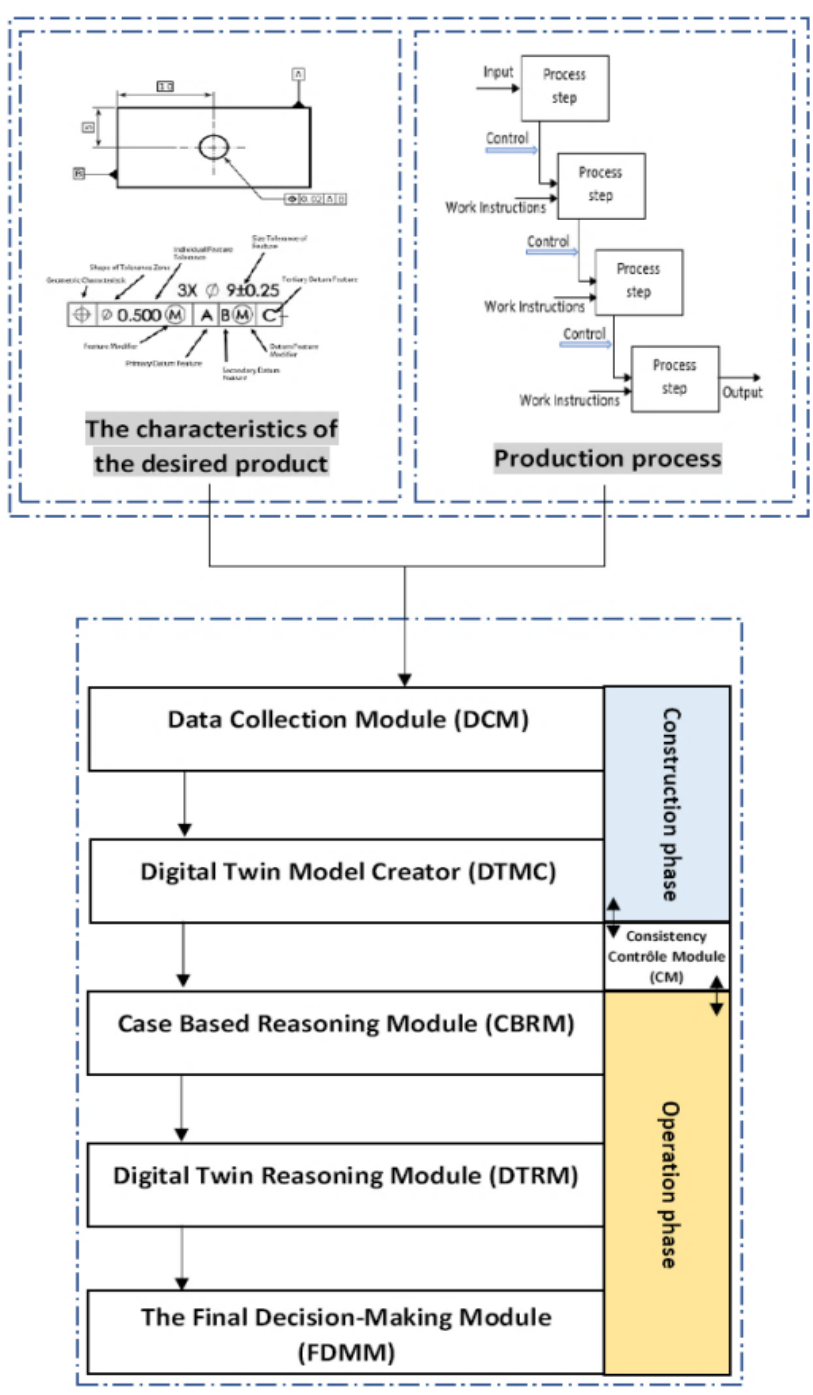

Fig. 1. The Global Structure of the Proposed Automated System for Selection of Production Parameters.

- The Digital Twin Model Creator (DTMC): It is the last module in construction phase and through which the digital twin model of the desired study is established. Indeed, five dimensions are included in this model, namely physical entity (PE), virtual model (VE), connection between PE and VE (CN), DT data (DD) and services (Ss) [13]. The physical entity will be simulated virtually, and the cyber-physical connection will be established based on a manufacturing ontology. In fact, the interoperability between the physical entity and the virtual model will be ensured throughout our DTM-Onto.

While the second phase (operation phase) contains three modules:

- The Case Based Reasoning Module (CBRM): The utility of this module is to understand and solve new problems out of old experiences. To do so, a reasoner recalls and uses a previous case, saved in a database and similar to the actual one to solve this new problem. 
Then, the reasoner analyzes the new situation, and tries either to adapt the already existing solutions to the problem, to create an equitable solution to solve it, or to interpret and to critique new solutions.

- The Digital Twin Reasoning Module (DTRM): Before manufacturing a product, the design team requires well determined dimensions and specifications. However, there are always deviations between the design and the manufactured product due to several criteria (production parameters, manufacturing tools, ...). Hence the importance of this module (DTRM) which plays the role of a second reasoner via the manufacturing ontology previously established. Thus, through the formalization of a set of queries, the ontology will provide direct answers to the production team on the production parameters to be configured at the level of physical resources. These queries will be formalized and used to ask the ontology for the optimal values of the production parameters to configure.

- The Final Decision-Making Module (FDMM): redoing an experiment represents a waste of time and money for companies. This is why this module is added to the methodology in order to save and archive the results obtained in previous situations, in data bases, to enrich the manufacturing ontology and to benefit from them in future experiments.

- The Consistency Control module (CCM): This module is responsible for controlling the consistency between the modules of the construction phase and those of the operation phase.

Fig. 2 shows the working process of the proposed SPPDT system.

In fact, the process starts with the collection of necessary information about the production process and the product, including its dimensions and specifications, as well as the number of blocks in the process and their functioning. After that, if the production process is composed of several blocks, it will be divided into many sections; when the production parameters change, the section changes, and then a section may contain one or more blocks. Subsequently, for each section, all possible combinations of production parameters (Xi) will be determined. The Digital Twin of the product is established for the first combination $\mathrm{X}_{1}$ and the CBR based decision making process is executed. If, the established Digital Twin model does not exist in the case base, the Digital Twin based reasoning process will be executed, otherwise this step will be ignored. In addition, the database is updated by saving and archiving the results obtained and deleting the repeated cases.

All the steps applied for the $\mathrm{X}_{1}$ combination are repeated for all the other combinations. Finally, the process ends with the determination of the optimal combination of production parameters. The modules of the proposed system will be detailed in the next sections.

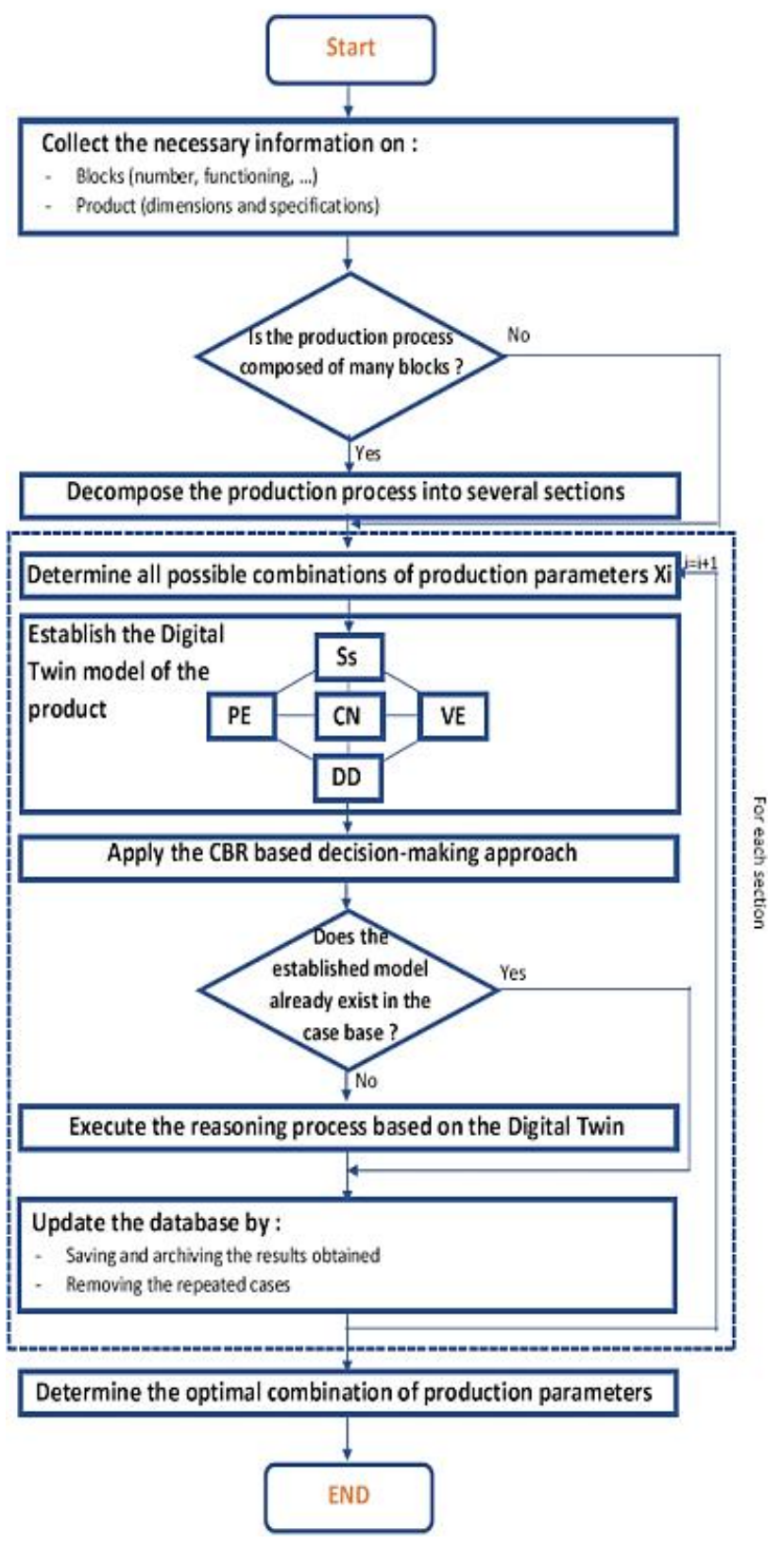

Fig. 2. The Working Process of the SPPDT System.

\section{Construction of the Digital Twin Model}

In this section, the digital twin model of the desired study is established by going through several steps:

\section{A. Step 1 : Define the Constraints of the Product}

The product constraints are decomposed into [21]:

- Dimensional constraints: which can be used to designate the size of specific entities or the relative location between different entities.

- Geometric constraints: which represent relations between geometric entities such as tangency, collinearity, parallelism, perpendicularity, coincidence of points, symmetry, etc. 
In this part, geometric and dimensional constraints are defined using the following notations:

- 1 : Number of product surfaces;

- $\mathrm{m}$ : Number of dimensional constraints in the surface $\mathrm{k}$ of the product;

- $\operatorname{cd}_{\mathrm{kj}}$ : Dimensional constraint, $\mathrm{k}=1, \ldots, 1$ and $\mathrm{j}=1, \ldots, \mathrm{m}$;

- $\mathrm{n}$ : Number of geometric constraints in the surface $\mathrm{k}$ of the product;

- $\quad \operatorname{cg}_{\mathrm{kj}}$ : Geometric constraint, $\mathrm{k}=1, \ldots, 1$ and $\mathrm{j}=1, \ldots, \mathrm{n}$.

For dimensional constraints, the measurements cannot be exact in reality, so for each of them a tolerance interval will be defined.

Concerning the geometrical constraints, the types of those existing will be determined for all surfaces (tangency, collinearity, etc.).

\section{B. Step 2 : Determine the Possible Combinations of \\ Production Parameters}

The majority of production processes consist of several blocks. These blocks will be grouped into several sections (n sections). Indeed, the section changes when the production parameters change from one block to another.

As shown in Fig. 3, each section contains $\mathrm{N}$ parameters to be set $(\mathrm{P} 1, \mathrm{P} 2, \ldots, \mathrm{PN})$ and each parameter can take multiple values $(\mathrm{A}, \mathrm{B}, \ldots, \mathrm{Z})$.

In addition to that, it should be noted that the production parameters and their numbers can be not the same from one section to another.

For example, P1 of section 1 is not necessarily the same P1 of section 2 .

Thereafter, $\alpha_{\mathrm{i}}=\mathrm{A} * \mathrm{~B} * \ldots * \mathrm{Z}$ combinations of production parameters can be generated for the $\mathrm{i}^{\text {th }}$ section, and these combinations are denoted $\mathrm{X}_{\mathrm{ij}}$.

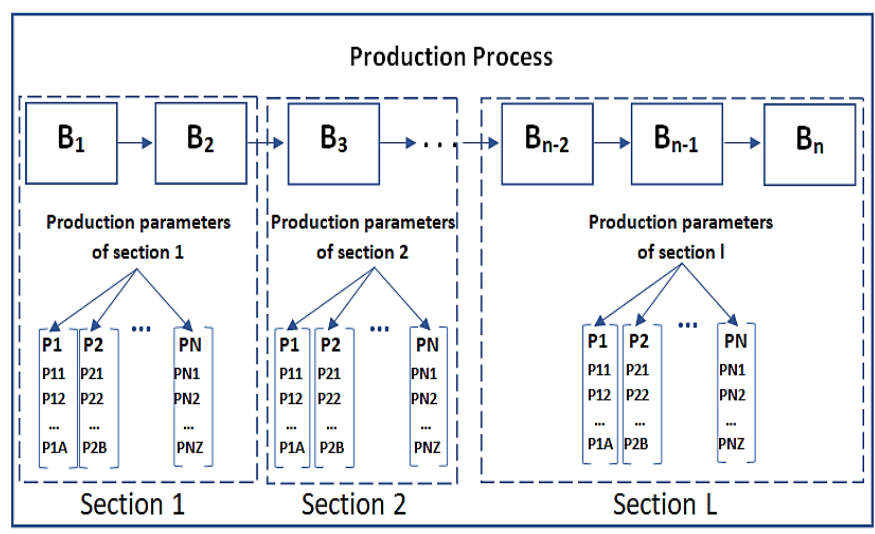

Fig. 3. The Notation used for Indexing Production Parameters.
- With: $\mathrm{i}=1, \ldots, \mathrm{L}$ and $\mathrm{j}=1, \ldots, \alpha$

- For example, $\mathrm{X}_{11}=\{\mathrm{P} 11, \mathrm{P} 21, \ldots, \mathrm{PN} 1\}$.

\section{Step 3 : Define the Digital Twin Model}

Once the product constraints and production parameters have been determined, all that remains is to establish the Digital Twin model of the production process. In fact, the decomposition of the whole process in multiple sections imposes the establishment of several sub-models, which will be gathered later, in order to obtain the global model of the Digital Twin.

The Digital Twin sub-model of each section will be represented as follows:

$\mathrm{DT}_{\mathrm{i}}=\left\{\mathrm{PE}_{\mathrm{i}}, \mathrm{VE}_{\mathrm{i}}, \mathrm{Ss}_{\mathrm{i}}, \mathrm{DD}_{\mathrm{i}}, \mathrm{CN}_{\mathrm{i}}\right\}$

With:

$\mathrm{i}=1, \ldots, \mathrm{L}$;

$\mathrm{L}$ is the number of the sections.

And then, the global model of the Digital Twin of the whole process will be as follows:

$\mathrm{DT}=\bigcup_{i=1}^{L} D T_{i}=\bigcup_{i=1}^{L}\left\{\mathrm{PE}_{\mathrm{i}}, \mathrm{VE}_{\mathrm{i}}, \mathrm{Ss}_{\mathrm{i}}, \mathrm{DD}_{\mathrm{i}}, \mathrm{CN}_{\mathrm{i}}\right\}$

It should be noted that the connection interface between the physical entity and the virtual model, as well as the Digital Twin Data Model and services are established based on an manufacturing domain ontology created and named "Digital Twin Manufacturing Ontology" (DTM-Onto). This ontology will be developed more in the part of the DTR module.

In this section, the DTM-Onto is constructed for two reasons. On one hand, this ontology will be the connection interface between the physical entity and the virtual model, as well as the data model and the Digital Twin services. On the other hand, the DTM-Onto will be used later to do the reasoning at the Levels of the CBR and DTR modules.

In this article, the DTM-Onto is developed in the ontology editor "Protégé". It is composed of three main elements which are [22,23]:

- Classes: are a set of individuals that describe concepts in a specific domain. In this paper, the classes are related to the elements in the manufacturing domain;

- Object properties: They identify the links between the classes and the individuals;

- Data properties: They define modifiers for ontology classes or establish characteristics of the instances.

Fig. 4 represents the different classes and Object Properties configured on the constructed DTM-Onto. Data Properties will be configured at the case study level. 


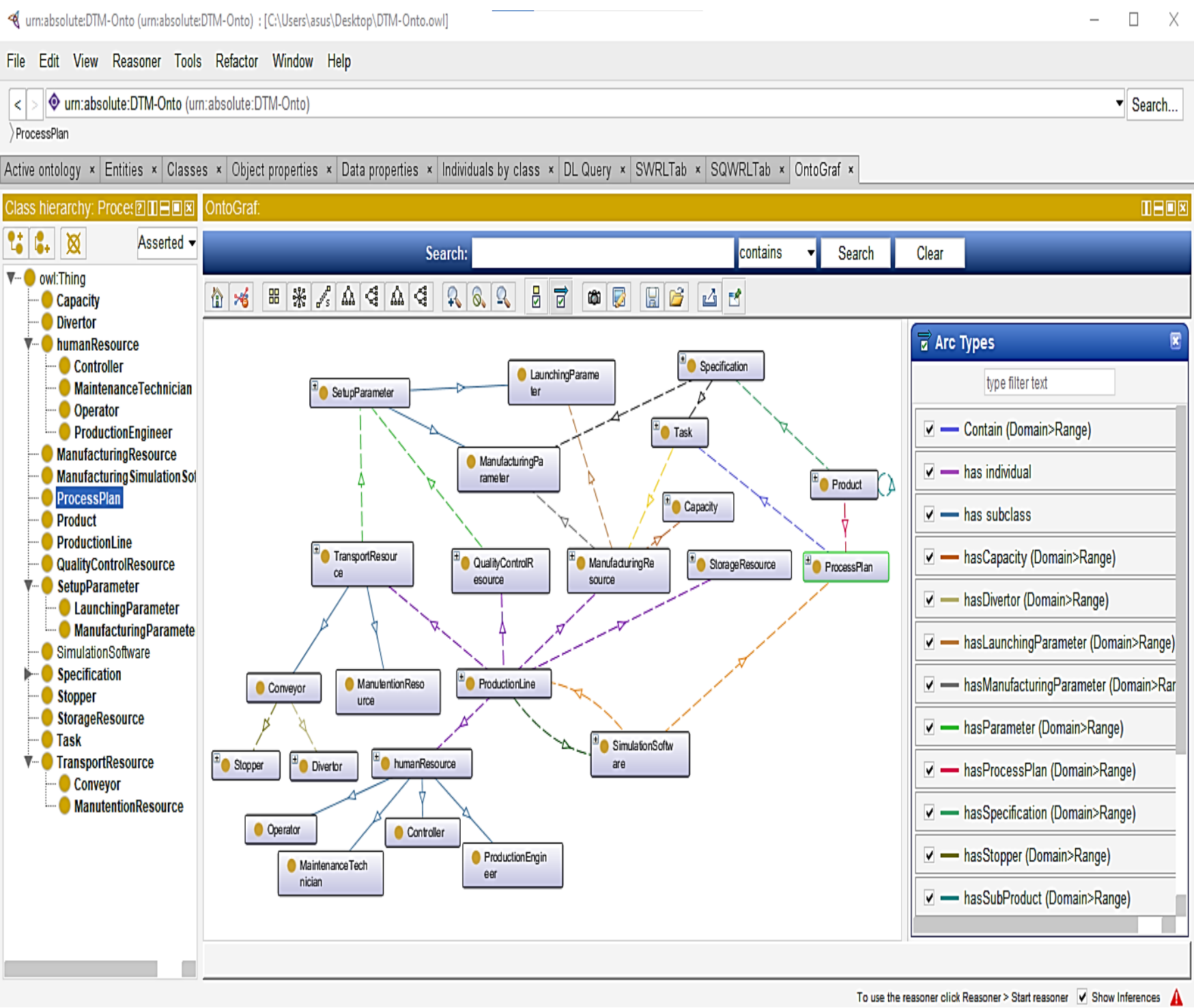

Fig. 4. The General Conceptual Model of the DTM-Onto Ontology Proposed.

\section{Description OF THE CASE-BASED REASONING (CBR)} MODULE FOR OPTIMAL CHOICE OF PRODUCTION PARAMETERS

The case-based reasoning module (CBR module) is based on the use of previous studies of production parameters choices, saved in databases in the company's information system. Thus, the old studies are used to benefit from their results for an optimal choice of production parameters for the new studied case.

As shown in Fig. 5, the proposed working process structure for the CBR module contains three main phases:

\section{A. Preliminary Phase}

First of all, an attribution of indexes to the cases is required to facilitate their retrieval. In fact, assume that the case base (CB) contains multiple problems $\left(\mathrm{C}_{\mathrm{i}}\right)$ which represent the specifications of the desired product, and their results which represent the appropriate production parameters for each section to realize it. Then, the case base can be represented as follows:
$\mathrm{CB}=\left\{\mathrm{C}_{1}, \mathrm{C}_{2}, \ldots, \mathrm{C}_{\mathrm{r}}\right\}$

With:

$r$ is the number of problems solved and stored in the case base.

Moreover, each case $\left(C_{i}\right)$ contains $L$ sub-cases $\left(C_{i j}\right)$. Each of these sub-cases is composed of two parts: the first one contains the data (production parameters) and the second one contains the generated results (product specifications):

$C_{i j}: \quad X_{i j}$ Results $\underset{\sum \mathrm{cd}_{\mathrm{kj}}}{\longrightarrow}+\sum \mathrm{cg}_{\mathrm{kj}}$

\section{B. Matching Phase}

In the matching phase, a comparison between what is desired and what already exists on the basis of cases will be done. In other words, a similarity index between the specifications of the products saved in the case base and those desired in the studied one will be calculated for each section. 


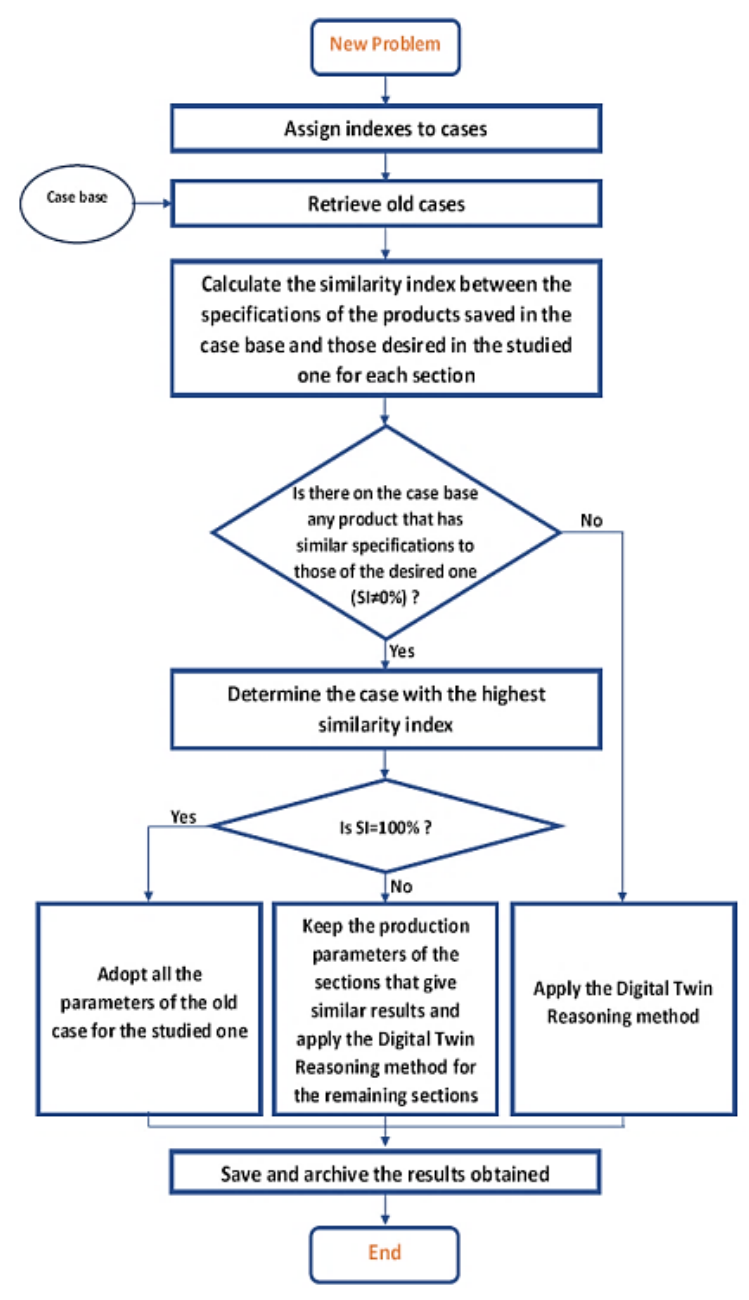

Fig. 5. The Proposed Working Process Structure for the CBR Module.

To do that, the working process shown in Fig. 6 will be executed, with:

- $\quad$ SI: the similarity index $(\%)$;

- $\mathrm{Cd}_{\mathrm{i}}$ : the set of dimensional constraints existing at the section $\mathrm{i}$;

- $\widetilde{\mathrm{Cd}_{1}}$ : the set of desired dimensional constraints in section i;

- $\mathrm{Cg}_{\mathrm{i}}$ : the set of geometrical constraints existing at the section $\mathrm{i}$;

- $\widetilde{\mathrm{Cg}_{1}}$ : The set of desired geometrical constraints in section i.

At the beginning of the process, "a" is equal to 0 and " $i$ " is equal to 1 .

It is clear that in each section a set of dimensional and geometric constraints of the product is realized. So at the beginning, a comparison at the level of section 1 will be made between the dimensional and geometrical constraints wanted on the new product and each old case existing in relation with Section 1: comparison between $\widetilde{\mathrm{Cd}_{1}}$ and each $\mathrm{Cd}_{1}$ as well as $\widetilde{\mathrm{Cg}_{1}}$ and each $\mathrm{Cg}_{1}$.

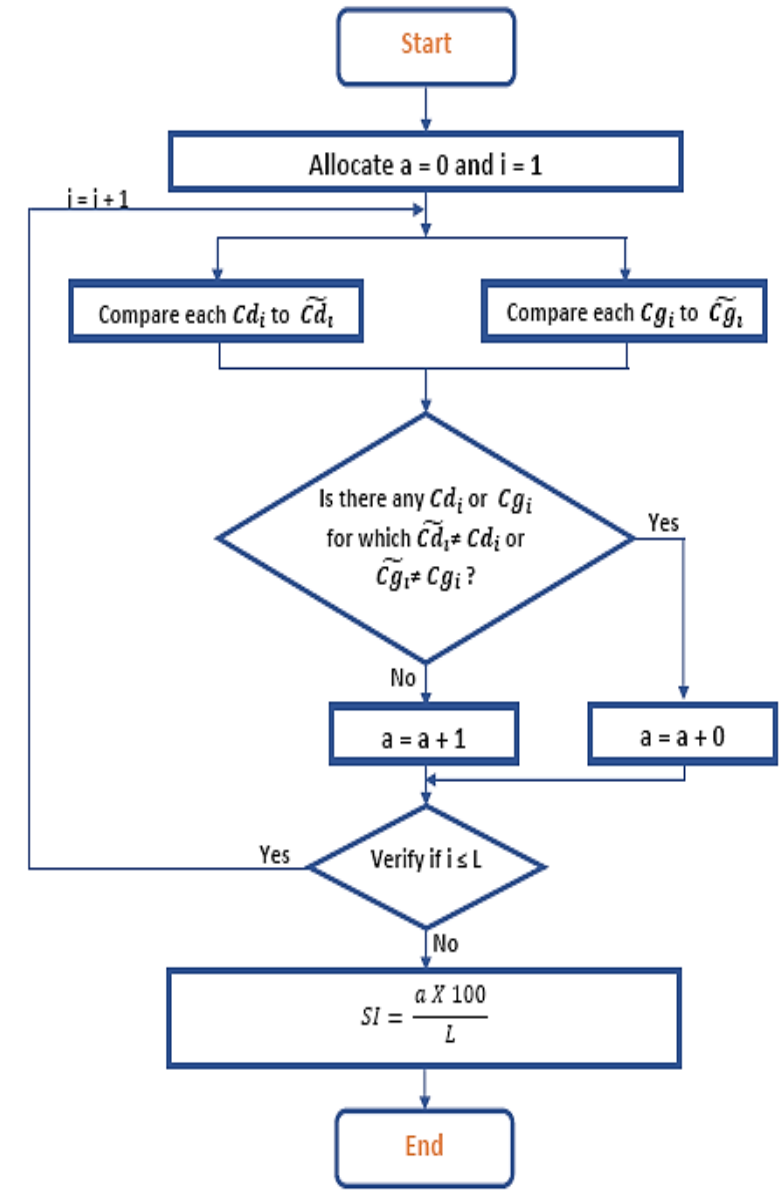

Fig. 6. The Algorithm for Calculating the Similarity Index.

After that, two alternatives can be considered:

- If there is an old product with the same geometrical and dimensional specifications as the new product, a will be incremented by 1 .

- If the new value of a is strictly superior to $\mathrm{L}$ (the total number of sections of the production process), the comparison cycle is interrupted immediately;

- If not, the comparison cycle will be executed for the next section.

- Otherwise, the comparison cycle is interrupted immediately.

Once the comparison cycle is completed, the similarity index is calculated by the following relation and the process is stopped:

$S I=\frac{a \times 100}{L}$

\section{Decision-Making Phase}

This is the most important phase of the CBR process.

Indeed, after the calculation of the similarity index in the matching phase, three cases are supposed to have: 
- $\mathrm{SI}=0 \%$ : the Digital Twin Reasoning method will be applied;

- $0 \%<\mathrm{SI}<100 \%$ : the production parameters of the sections that give similar results will be kept and the Digital Twin Reasoning method will be applied for the remaining sections;

- $\mathrm{SI}=100 \%$ : all the parameters of the different sections of the previous similar cases will be adopted for the studied one.

Finally, the new results obtained will be saved and archived in the case database for a probable benefit in the realization of new products.

\section{DESCRIPTION OF THE Digital Twin REASONING MODULE (DTRM) FOR OPTIMAL CHOICE OF PRODUCTION PARAMETERS}

The Digital Twin Reasoning Module (DTRM) is very important in complementing the proposed SPPDT methodology.

The backbone of this module consists of the manufacturing domain ontology (DTM-Onto, which plays the role of connection interface between the physical entities and the virtual entities of the Digital Twin developed before. And due to its reasoning capacities, the DTM-Onto will be used to automate the calculation of production parameters as it will be described later.

Fig. 7 shows the operating system of the DTR module.

The first step will be to formalize the business rules related to each manufacturing process using the SWRL (Semantic Web Rule Language) and to introduce them in the DTM-Onto ontology. For example, for the stamping process, the formalized business rules will allow the calculation of the falling mass of the press to be used, the number of punches required to obtain all the geometric details of the product, the speed of the punches, the punching force, etc. For the machining process, the formalized business rules will allow the calculation of the feed rates, the number of passes, etc.

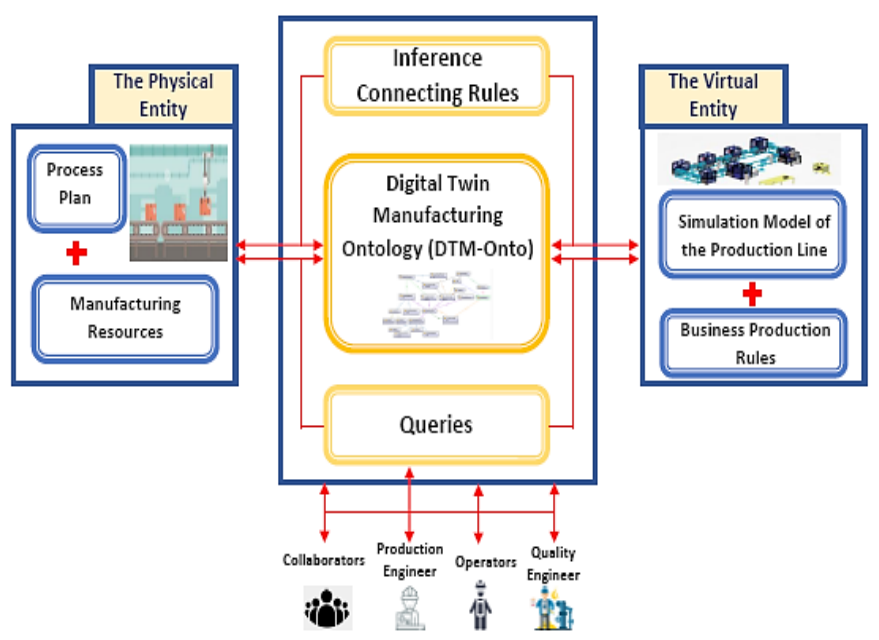

Fig. 7. The Operating System of the DTR Module.
Simulations using flow modeling software such as WITNESS, 3DEXPERIENCE, etc., will be used to validate the production parameters to be used and to virtually visualize the production sequences. Conveyor speeds as well as Pick and Place programs for handling robots will also be determined during this phase.

Once all the production parameters are validated by the simulation, the DTM-Onto ontology will be enriched with them. Indeed, as it will be described in the following, instances and object properties will be added to DTM-Onto to do so.

A second category of inference rules will then be executed. These are the matching rules between the production parameters of the virtual entities and those of the physical entities. These rules will ensure the interoperability at the virtual/physical interface of the DT in a dynamic way.

Finally, through the formalization of a set of queries, the ontology will provide direct answers to the production team on the production parameters to be configured at the physical resource level. These queries will be formalized using the SQWRL (Semantic Query-Enhanced Web Rule Language) and used to ask the ontology about the appropriate production parameters.

\section{CASE STUDY}

In this section, an industrial case study is presented to validate the operation and applicability of the proposed SPPDT methodology.

Indeed, the various phases of the proposed methodology are applied, in this section, on the production process of yogurt in a company specialized in manufacturing dairy products.

Initially, the company manufactures small yogurt cups with the geometric and dimensional specifications shown in Fig. 8 and Table I.

To do so, the realization of the final product requires two main parts, namely: a PROCESS part and a conditioning part, but the case study will only focus on the conditioning part.

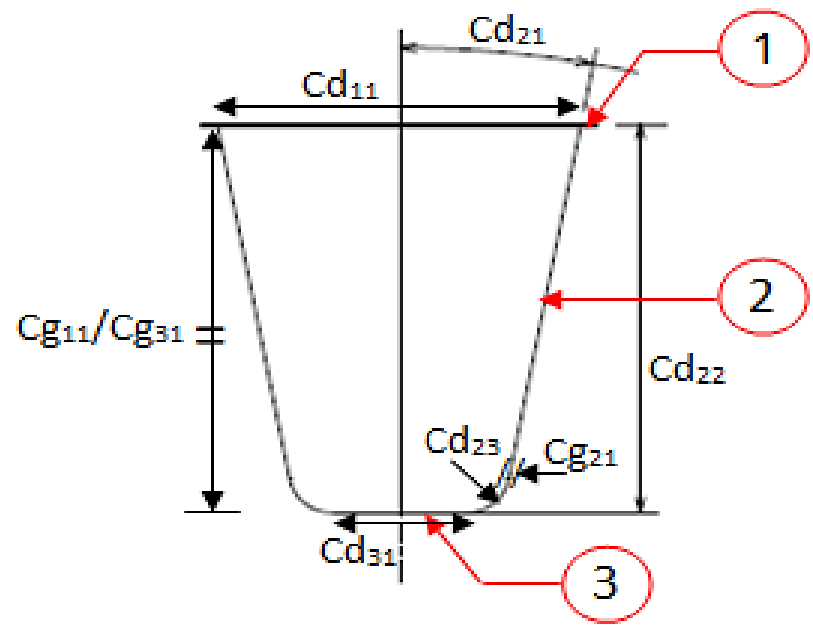

Fig. 8. Indexing of Geometrical and Dimensional Constraints of the Product. 
TABLE I. THE DIMENSIONAL AND GEOMETRICAL CONSTRAINTS OF THE Two CUP SIZES

\begin{tabular}{|l|l|l|}
\hline $\begin{array}{l}\text { Dimensional and geometrical } \\
\text { constraints }\end{array}$ & Small cups & Large cups \\
\hline $\boldsymbol{C d}_{11}$ & $70 \mathrm{~mm}$ & $80 \mathrm{~mm}$ \\
\hline $\boldsymbol{C d}_{21}$ & $10^{\circ}$ & $10^{\circ}$ \\
\hline $\boldsymbol{C d}_{22}$ & $70 \mathrm{~mm}$ & $95 \mathrm{~mm}$ \\
\hline $\boldsymbol{C d}_{23}$ & $10 \mathrm{~mm}$ & $10 \mathrm{~mm}$ \\
\hline $\boldsymbol{C d}_{31}$ & $10.5 \mathrm{~mm}$ & $10.5 \mathrm{~mm}$ \\
\hline $\boldsymbol{C g}_{21}$ & Tangency & Tangency \\
\hline $\boldsymbol{C g}_{22}$ & Symmetry & Symmetry \\
\hline $\boldsymbol{C g}_{11} / \boldsymbol{C g}_{31}$ & Parallelism & Parallelism \\
\hline
\end{tabular}

This phase is realized on a production line which is composed of several blocks that allow executing a set of operations.

In fact, after unrolling the plastic strip (PS), a heating system consisting mainly of heating resistances and temperature probes (for continuous regulation) allows the heating of the PS edges. This operation facilitates its pecking and thus its transport throughout the conditioning process. This transfer is carried out by means of a pimple chain.

Afterwards, an ionizing deduster removes any foreign matter from the PS.

Immediately after, a heating box driven by a cam press is installed. Its role is to heat the PS surfaces which will undergo a deformation. The next step is to form the yogurt pots in the form of packs of 24 pots (the plastic forming block) and to dose them with a piston doser. At the same time, a polymix unwinder allows, as its name indicates, to unroll the polymix with the help of an automatic splicing system. This system allows changing the reel of the polymix automatically. In turn, the polymix passes through a tunnel of UV lamps allowing its ionization so that a dating can take place afterwards.

Before the yogurt pots are cut into packs (24 pots), the polymix is welded to the already dosed pots in a welding block which is also driven by a cam press.

Once the packs are cut, they are packed in plastic boxes. Their transfer from the sealing block to the case packer is done using a conveyor called pilger conveyor.

To finish the conditioning of the yogurt, the full cases are palletized manually on wooden pallets and then stored in cold rooms.

After a study of the market, the company decided to start manufacturing large size cups. These cups are different from the small ones by their dimensional constraints as it is represented in Fig. 8.

This change of series had a big problem of pots piercing during their production. After the analysis of the major causes of the appearance of this defect, it was shown that the problem comes from the bad adjustment of the parameters of production.
Fig. 9 shows the problem of piercing cups.

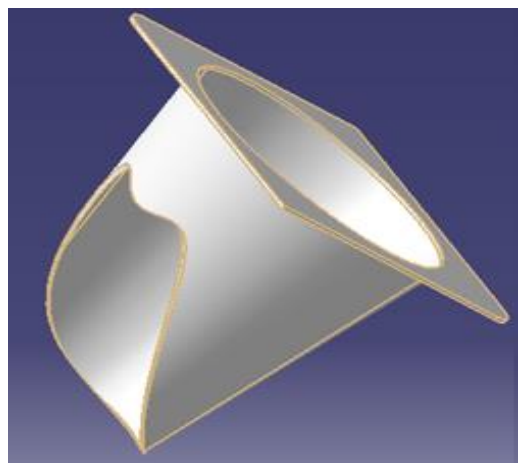

Fig. 9. The Problem of Cups Piercing.

We remind that there are three categories of production parameters to consider. The first one concerns the manufacturing process itself (for the forming of the cups: the heating temperature, the pressure, the depth of the punches,...). The second category concerns the product specifications (dimensional and geometrical constraints of the cups). While the third class concerns food safety (sterilization time, sterilization temperature,...).

This last class of parameters has not been taken into account in the case of study because it respects the food safety standards, and therefore there are no parameters to choose because they are already imposed by the standards.

So, to solve this parameters adjustment problem, the SPPDT approach is executed.

The first step is to place several sensors on the production process in order to copy virtually the physical state of its components.

Fig. 10 shows an extract from the virtual representation of the production process.

In addition to that, the DTM-Onto which plays the role of interoperability interface between the physical entity and its virtual model is enriched with the necessary data for its efficient functioning, namely: the product specifications, the different blocks of the process, the manufacturing parameters, etc.

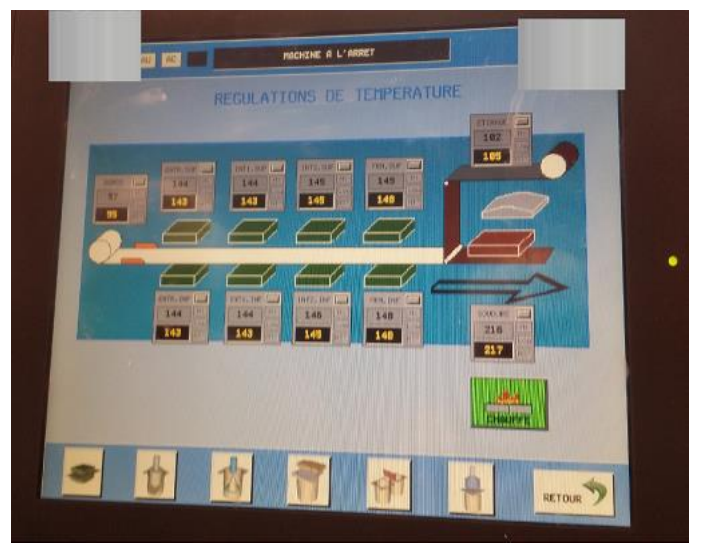

Fig. 10. An Extract from the Virtual Representation of the Production Process. 
In the following, the rest of the SPPDT approach is executed on the production process block by block.

For the plastic unrolling block, the first step is to execute the CBR. To do so, the algorithm for calculating the similarity index is applied to the initial product (the raw material: the plastic strip). In our case, the specifications of the plastic strip (material, length, width) used for the manufacture of the large cups are the same as the small ones, the difference that exists is only its thickness, and then: $0 \%<\mathrm{SI}<100 \%$. This result sends us directly to the use of the Digital Twin Reasoning Module.

This step consists in formalizing a SWRL rule of conservation of the volume between the pot and its raw material. So:

Volume (cup) $=$ Volume $($ PS)

$\Rightarrow$ Volume $($ cup $)=$ Length $(\mathrm{PS}) *$ Width $(\mathrm{PS}) *$ Thickness $(\mathrm{PS})$

$\Rightarrow$ Thickness $(\mathrm{PS})=\frac{\text { Volume }(\text { cup })}{\text { Length }(\mathrm{PS}) * \text { Width }(\mathrm{PS})}$

And thus the formalized rule is the rule S3 of the Table II.

Fig. 11 shows the plastic strip thickness calculation SWRL rule that we encoded in the SWRL tab of Protégé5.0, its results and their explication that we generated after running the reasoning with the Pellet reasoner.

The same steps applied on the unrolling block are applied on the heating box. The plastic strip introduced into this block has the same specifications (material, length and width) as the one used for the manufacture of small cups, except its thickness which changes, and it is heated to a temperature between the fusion limit and the elasticity limit of the polymer. Consequently: $0 \%<\mathrm{SI}<100 \%$ and the transition to the DTR module is crucial.

This heating temperature is calculated empirically by the following formula:

$T_{\text {heating }}^{\circ}=T_{\text {fusion }}^{\circ}-0.7 *\left(T_{\text {fusion }}^{\circ}-T_{\text {elasticity }}^{\circ}\right)$

This rule is formalized in SWRL by the rule S2 in the Table II, at the DTM-Onto level to automate its calculation. Fig. 12 shows the heating temperature calculation rule encoded in Protégé 5.0, its results and their explanation.

After this, the CBR algorithm is executed on the plastic forming block. In fact, there have been considerable changes in the dimensional and geometric specifications of the new cups that will be manufactured, and subsequently: $0 \%<\mathrm{SI}<100 \%$ and the transition to DTR is mandatory.

What validates the proposed approach is that empirically, when the same production parameters (notably the same punch and the same depth of pass) are kept for the two types of pots, certain non-conformities appear in the product. So to solve this problem and automate the selection of optimal production parameters to use, the DTR is executed. Indeed, three main categories of business rules are defined in the SWRL tab:
- Category 1 (R1-R5 and R9): Formalizes the correspondences between the dimensional specifications of the punches used and the cups formed.

- Category 2 (R6-R8): Formalizes the correspondence between the geometric specifications of the punches used and the cups formed.

- Category 3 (S1): represents the rule of calculation of the depth of descent of the punches. The descent speed remains the same.

TABLE II. BUSINESS RULES PROGRAMMED ON THE ONTOLOGY

\begin{tabular}{|l|}
\hline Business rules \\
\hline R1: Product $(? Y)^{\wedge}$ hasProductDimensionalConstraint_Cd11(?Y, ?a ${ }^{\wedge}$ \\
isAssociatedTo_Punch(?Y, ?P) \\
$->$ hasPunchDimensionalConstraint_Cd11(?P, ?a)
\end{tabular}

R2 : Product(?Y)^ hasProductDimensionalConstraint_Cd21(?Y, ?a)^

isAssociatedTo_Punch(?Y, ?P)

-> hasPunchDimensionalConstraint_Cd21(?P, ?a)

R3 : Product(?Y)^ hasProductDimensionalConstraint_Cd22(?Y, ?a)^

isAssociatedTo_Punch(?Y, ?P)

-> hasPunchDimensionalConstraint_Cd22(?P, ?a)

R4 : Product(?Y)^ hasProductDimensionalConstraint_Cd23(?Y, ?a)^

isAssociatedTo_Punch(?Y, ?P)

-> hasPunchDimensionalConstraint_Cd23(?P, ?a)

R5 : Product(?Y) ^ hasProductDimensionalConstraint_Cd23(?Y, ?a)^

isAssociatedTo_Punch(?Y, ?P)

-> hasPunchDimensionalConstraint_Cd23(?P, ?a)

R6 : Product(?Y)^ hasProductGeometricalConstraint_Cg11(?Y, ?a)^

isAssociatedTo_Punch(?Y, ?P)

-> hasPunchGeometricalConstraint_Cg11(?P, ?a)

R7 : Product(?Y)^ hasProductGeometricalConstraint_Cg21(?Y, ?a)^

isAssociatedTo_Punch(?Y, ?P)

-> hasPunchGeometricalConstraint_Cg21(?P, ?a)

R8 : Product(?Y)^ hasProductGeometricalConstraint_Cg22(?Y, ?a)^

isAssociatedTo_Punch(?Y, ?P)

$\rightarrow$ hasPunchGeometricalConstraint_Cg22(?P, ?a)

R9 : Product(?Y)^^ hasProductDimensionalConstraint_Cd31(?Y, ?a)^ isAssociatedTo_Punch(?Y, ?P)

-> hasPunchDimensionalConstraint_Cd31(?P, ?a)

S1 : Product(?q)^ hasProductDimensionalConstraint_Cd22(?q, ?i) ^

Punch(?P)^isAssociatedTo_Punch(?q, ?P)

-> hasPassingDepth(?P, ?i)

S2 : Product(?q)^ hasRawMaterial(?q, ?M)^ hasFusionTemperature(?M, ?f

${ }^{\wedge}$ hasElasticLimitTemperature(?M, ?e $\wedge^{\wedge} \operatorname{swrlb}$ :subtract(?a, ?f, ?e $)^{\wedge}$

swrlb:multiply(?b, ?a, -0.7)^ swrlb:add(?c, ?b, ?f) ->

hasFormingTemperature(?q, ?c)

S3 : Container( ?q $)^{\wedge} \operatorname{RawMaterial}(? \mathrm{p})^{\wedge}$ isConstructedFrom_Plate(?q,?p $)^{\wedge}$

hasVolume_in_mm3 $(? \mathrm{q}, ? \mathrm{~V})^{\wedge}$ hasLenght_in_mm $(? \mathrm{p}, ? \mathrm{j})^{\wedge}$ hasWidth_in_mm

$(? \mathrm{p}, ? \mathrm{w})^{\wedge}$ swrlb:multiply $(? \mathrm{~h}, ? \mathrm{j}, ? \mathrm{w})^{\wedge}$ swrlb:divide $(? \mathrm{i}, ? \mathrm{~V}, ? \mathrm{~h})$

$\rightarrow$ hasThickness_in_mm (?q,?i) 


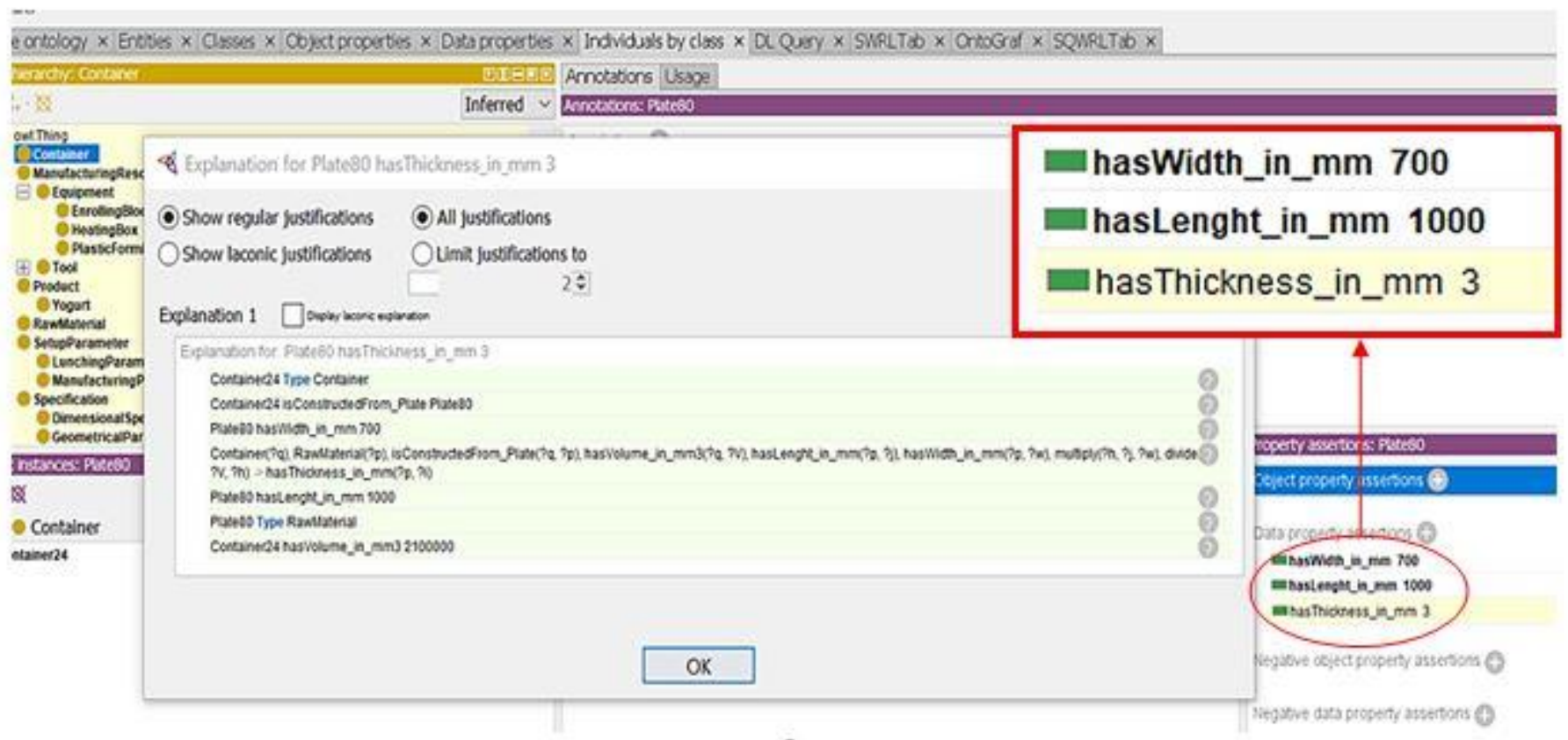

Fig. 11. The Plastic Strip Thickness Calculation Rule Encoded in Protégé 5.0, its Results and their Explanation.

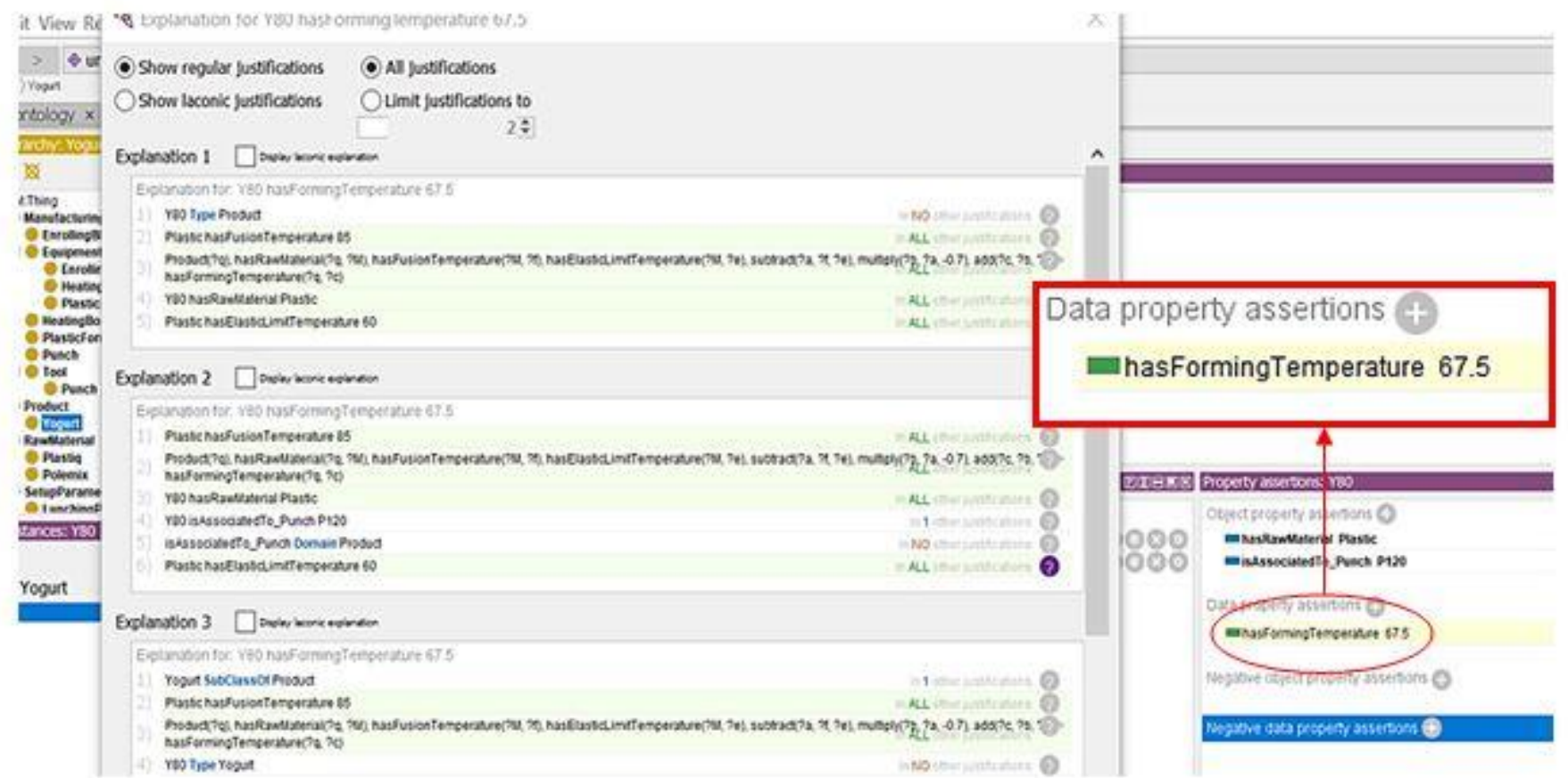

Fig. 12. The Heating Temperature Calculation Rule Encoded in Protégé 5.0, its Results and their Explanation.

All these rules, their results and explanations are shown in Fig. 13. All these rules, their results and explanations are shown in Fig. 13. For instance, the ontology concluded that the dimensional constraint $\mathrm{Cd} 31$ of the punch is $10.5 \mathrm{~mm}$.
For the remaining block, i.e. the welding block, the welding parameters are kept the same considering that the SI $=100 \%$ (the same manufacturing material).

From the results obtained, it is clear that the SPPDT approach has given very satisfying results and therefore it can be validated. 


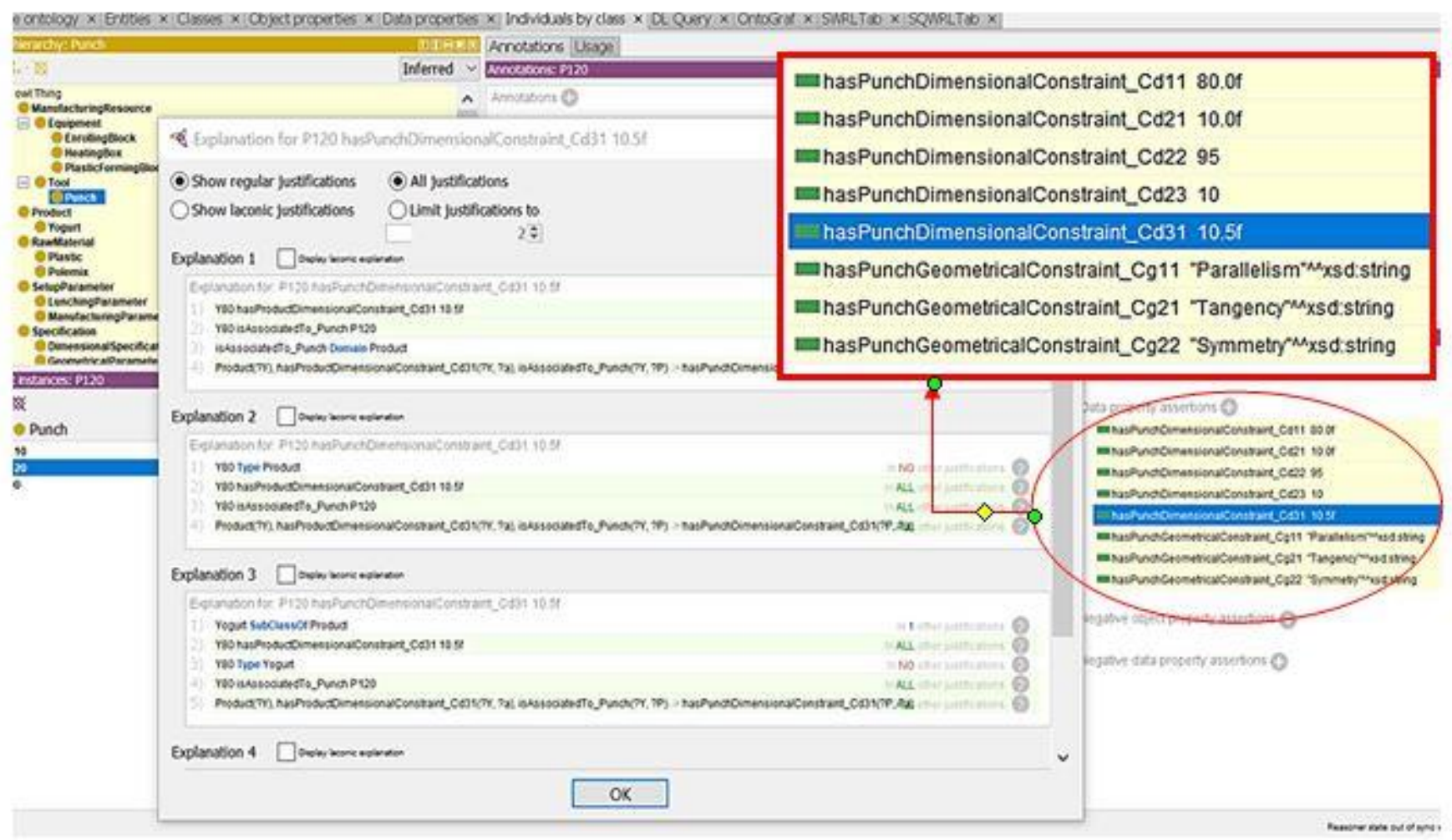

Fig. 13. The Plastic Forming Block Rules Encoded in Protégé 5.0, their Results and Explanations.

\section{CONCLUSION AND PERSPECTIVES}

In this paper, a new automated artificial intelligence system is developed to support decision making for selection of production parameters. Its originality lies in the integration, for the first time, of three different artificial intelligence tools, namely: digital twin, ontologies and case-based reasoning. The integration of these tools in a flexible hybrid system allows benefiting from the different advantages of each of them. In fact, the digital twin allowed us to simulate the production processes and their parameters in real time, as well as to validate the production parameters on the virtual production processes before their physical implementation. On the other hand, the use of ontologies allowed us to ensure the interoperability between the different elements of the cyberphysical model of the digital twin, to ensure the expressiveness of the treated information and to preserve their semantics. In addition to that, ontologies and together with CBR ensured reasoning and decision making for the selection of optimal production parameters. Differently from previous works which always present connection limits between the physical and the virtual DT, we have dealt with a double problematic, that of reasoning for the optimal choice of the production parameters, and that of interoperability via the assurance of the physicalvirtual connection.

The originality of this work also lies in the efficiency of our ontology to be adapted to several domains, through the formalization of business rules.

To illustrate these advantages and the effectiveness of the developed SPPDT system, an industrial case study is presented at the end of this document. Indeed, the development of this study allowed us to select the optimal production parameters of the studied process in an automated way.

As perspectives, it is suggested to enrich the developed SPPDT system, in particular its DTM-Onto, by integrating other aspects such as the degradation of the equipment, the external factors influencing the production, the safety of the equipment and the personnel, etc. Another perspective is to process and automate the selection of production parameters using other artificial intelligence tools.

\section{REFERENCES}

[1] G. Mezzour, S. Benhadou, and H. Medromi, "Digital Twins Development Architectures and Deployment Technologies: Moroccan use Case", International Journal of Advanced Computer Science and Applications (IJACSA), vol. 11, no. 2, 2020.

[2] L. D. Xu, E. L. Xu, and L. Li, "Industry 4.0: state of the art and future trends", International Journal of Production Research, vol. 56, no. 8, p. 2941-2962, Apr. 2018.

[3] Z. Cunbo, J. Liu, and H. Xiong, "Digital twin-based smart production management and control framework for the complex product assembly shop-floor", The international journal of advanced manufacturing technology, vol. 96, no. 1-4, p.1149-1163, 2018.

[4] M. Cuc, "Improving the decision-making process by modeling digital twins in a big data environment.", Management \& Marketing Journal, vol.19, no.1, 2021.

[5] B. Hamrouni, A. Bourouis, A. Korichi et al., "Explainable OntologyBased Intelligent Decision Support System for Business Model Design and Sustainability", Sustainability, vol. 13, no. 17, p. 9819, 2021.

[6] A. Martin, S. Emmenegger, K. Hinkelmann \& B. Thönssen, "A viewpoint-based case-based reasoning approach utilising an enterprise architecture ontology for experience management", Enterprise Information Systems, 11(4), p. 551-575, 2017. 
[7] T. R. Gruber, "Towards Principles for the Design of Ontologies Used for Knowledge Sharing in Formal Ontology in Conceptual Analysis and Knowledge Representation", Kluwer Academic Publishers, 1993.

[8] R. Gámez Díaz, "Digital Twin Coaching for Edge Computing Using Deep Learning Based 2D Pose Estimation", Thèse de doctorat. Université d'Ottawa/University of Ottawa, 2021.

[9] M Grieves, "Virtually perfect: Driving Innovative and Lean Products Through Product Lifecycle management", Space Coast Press, Cocoa Beach, Fl. USA, 2011.

[10] M Grieves, J Vickers, "Digital twin: mitigating unpredictable, undesirable emergent behavior in complex systems", Transdisciplinary Perspectives on Complex Systems, Springer International Publishing, Berlin, Germany, 2017.

[11] F. Xiang, Z. Zhang, Y. Zuo et al., "Digital twin driven green material optimal-selection towards sustainable manufacturing". Procedia Cirp, vol. 81, p. 1290-1294, 2019.

[12] F. Tao, F. Sui, A. Liu et al., "Digital twin-driven product design framework", International Journal of Production Research, vol. 57, no. 12, p. 3935-3953, 2019.

[13] F. Tao, M. Zhang, Y. Liu et al., "Digital twin driven prognostics and health management for complex equipment". Cirp Annals, vol. 67, no 1, p. 169-172, 2018.

[14] International Standard Organization, «ISO-14258-1998: Systèmes d'automatisation industrielle, concepts et règles pour modèles d'entreprises» 1998.

[15] V. Fortineau, "Contribution à une modélisation ontologique des informations tout au long du cycle de vie du produit", Chemical and
Process Engineering, Ecole nationale supérieure d'arts et métiers ENSAM., France, 2013.

[16] G. Declerck, A. Baneyx, X. Aimé et al., " Les ontologies fondationnelles peuvent-elles débabeliser le web? ”, Rev. d'Intelligence Artif., vol. 28, no 2-3, p. 191-216, 2014.

[17] M. Garetti, L. Fumagalli and E. Negri, "Role of ontologies for CPS implementation in manufacturing", Management and Production Engineering Review, vol. 6, no. 4, p. 26-32, 2015.

[18] V. Fortineau, T. Paviot and S. Lamouri, "Improving the interoperability of industrial information systems with description logic-based models the state of the art", Computers in industry 64, p. 363-375, 2013.

[19] A. Abadi, H. Ben-azza et S. Sekkat, "An ontology-based support for knowledge modeling and Decision-Making in Collaborative Product Design", International Journal of Applied Engineering Research, vol. 12, no 16 , p. 5739-5759, 2017.

[20] A. Matsokis et D. Kiritsis, "An ontology-based approach for product lifecycle management", Computers in industry, 61, p. 787-797, 2010.

[21] F. Naya, M. Contero, J. Dorribo Camba et al., "On the role of geometric constraints to support design intent communication and model reusability", 2020.

[22] M. D. De Azevedo Jacyntho, et D. Morais, "Ontology-based decisionmaking", In : Web Semantics. Academic Press, p. 195-209, 2021.

[23] M. Horridge,S. Jupp, G. Moulton, A. Rector, R. Stevens and C. Wroe, "A practical guide to building owl ontologies using protégé 4 and co-ode tools edition1", vol. 2, The university of Manchester, pp. 107, 2009. 